\title{
Adaptive Rates of High-Spectral-Efficiency WDM/SDM Channels Using PDM-1024-QAM Probabilistic Shaping
}

Hu, Hao; Yankov, Metodi Plamenov; Da Ros, Francesco; Amma, Yoshimichi; Sasaki, Yusuke; Mizuno, Takayuki; Miyamoto, Yutaka; Galili, Michael; Forchhammer, Søren; Oxenløwe, Leif Katsuo Total number of authors: 11

Published in:

Proceedings of ECOC 2017

Publication date:

2017

Document Version

Peer reviewed version

Link back to DTU Orbit

Citation (APA):

Hu, H., Yankov, M. P., Da Ros, F., Amma, Y., Sasaki, Y., Mizuno, T., Miyamoto, Y., Galili, M., Forchhammer, S., Oxenløwe, L. K., \& Morioka, T. (2017). Adaptive Rates of High-Spectral-Efficiency WDM/SDM Channels Using PDM-1024-QAM Probabilistic Shaping. In Proceedings of ECOC 2017 IEEE.

\section{General rights}

Copyright and moral rights for the publications made accessible in the public portal are retained by the authors and/or other copyright owners and it is a condition of accessing publications that users recognise and abide by the legal requirements associated with these rights.

- Users may download and print one copy of any publication from the public portal for the purpose of private study or research.

- You may not further distribute the material or use it for any profit-making activity or commercial gain

- You may freely distribute the URL identifying the publication in the public portal 


\title{
Adaptive Rates of High-Spectral-Efficiency WDM/SDM Channels Using PDM-1024-QAM Probabilistic Shaping
}

\author{
Hao Hu ${ }^{(1)}$, Metodi Plamenov Yankov ${ }^{(1)}$, Francesco Da Ros ${ }^{(1)}$, Yoshimichi Amma ${ }^{(2)}$, Yusuke Sasaki( ${ }^{(2)}$, \\ Takayuki Mizuno $^{(3)}$, Yutaka Miyamoto ${ }^{(3)}$, Michael Galili $^{(1)}$, Søren Forchhammer ${ }^{(1)}$, Leif K. Oxenløwe ${ }^{(1)}$, \\ and Toshio Morioka ${ }^{(1)}$ \\ (1) DTU Fotonik, Department of Photonics Engineering, Technical University of Denmark, DK-2800, \\ Kgs. Lyngby, Denmark, huhao@fotonik.dtu.dk \\ (2) Advanced Technology Laboratory, Fujikura Ltd., Sakura, Chiba, 285-8550, Japan \\ ${ }^{(3)}$ NTT Network Innovation Laboratories, NTT Corporation, Hikarinooka, Yokosuka, Kanagawa, 239- \\ 0847, Japan
}

\begin{abstract}
We demonstrate adaptive rates and spectral efficiencies in WDM/SDM transmission using probabilistically shaped PDM-1024-QAM signals, achieving up to 7-Tbit/s data rates per spatial-superchannel and up to 297.8-bit/s/Hz aggregate spectral efficiency using a 30-core fiber on 12.5 and 25GHz WDM grids.
\end{abstract}

\section{Introduction}

To meet the ever-growing demands for higher bandwidth, the potential of space-division multiplexing (SDM) based on single-mode multicore fibers (SM-MCFs) or few-mode multi-core fibers (FM-MCFs) has been demonstrated in several impressive results ${ }^{1-3}$. SM-MCF transmission systems have the advantage of being free from mode-dependent loss/modal differential group delay and remove the need for high-complexity multiple-input multiple-output (MIMO) processing, lowering both power consumption and latency. In order to further increase the transmission capacity, dense SDM based on high-count SM-MCFs over 30 cores have been studied recently ${ }^{4}$. To further increase the data capacity in high-count SM-MCF transmission, more advanced modulation formats with higher spectral efficiency (SE) will be needed. While 1024-QAM and 2048-QAM have been used for single channel SM-MCF transmission with a symbol rate of $\sim 3 \mathrm{GBd}^{5,6}$, the most advanced modulation format used for WDM/SDM transmission so far is PDM-64$\mathrm{QAM}^{2,7}$. To achieve higher-order QAM (such as 1024-QAM) with higher symbol rates (>10 GBd) over multiple WDM channels, there are several

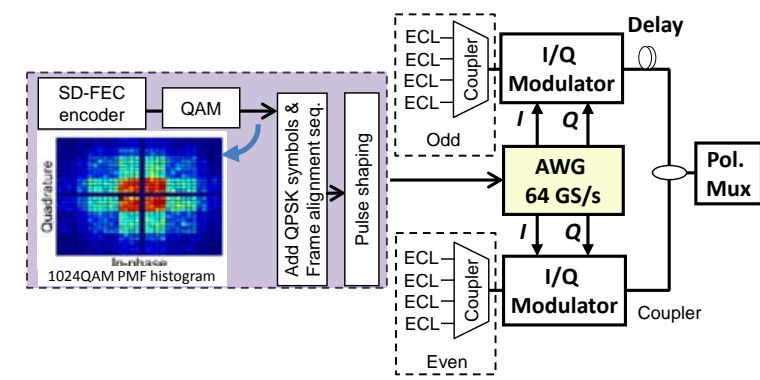

challenges such as hardware-limitations of available transceivers, achievable OSNR after transmission and the cost of narrow-linewidth lasers. Recently, probabilistic shaping (PS) has attracted a lot of attention in optical communications, not only for achieving shaping gain but also for the capability of adapting their rate to the channel conditions ${ }^{8-10}$.

In this paper, we demonstrate the first probabilistic shaping (PS) for WDM/SDM transmission. Eight WDM channels either modulated at $12 \mathrm{GBd}$ on a $12.5-\mathrm{GHz}$ grid or at 24.5 GBd on a $25-\mathrm{GHz}$ grid carrying PS PDM1024-QAM signals are transmitted through a 9.6-km 30-core fiber, reaching the highest modulation format order for WDM/SDM transmission to date. We employ full softdecision (SD)-FEC decoding with adaptive information rates for the different spectral and spatial channels to obtain error-free performance, and achieve aggregate data rates of 3.7 Tbit/s and $7 \mathrm{Tbit} / \mathrm{s}$ per frequency channel for the $12.5-\mathrm{GHz}$ grid and $25-\mathrm{GHz}$ grid cases, respectively. We thus achieve record-high spectral efficiencies of $297.8 \mathrm{bit} / \mathrm{s} / \mathrm{Hz}$ and 280.3 $\mathrm{bit} / \mathrm{s} / \mathrm{Hz}$ for the $12.5-\mathrm{GHz}$ grid and $25-\mathrm{GHz}$ grid cases, respectively, for SM-MCF transmission

Fig. 1: Schematic of experimental setup for WDM/SDM transmission of probabilistic shaped PDM-1024-QAM channels over a 9.6-km 30-core fiber, including external cavity lasers (ECLs) with a linewidth of $10 \mathrm{kHz}$, erbium-doped fibre amplifier (EDFA), arbitrary waveform generator (AWG), polarization multiplexer (Pol. Mux), optical bandpass filter (OBF). Inset: cross-sectional view of the 30-core fiber. 
systems.

\section{Experimental setup}

The experimental setup for the WDM/SDM transmission of PS PDM-1024-QAM channels is shown in Fig. 1. Eight external cavity lasers (ECLs) with a linewidth of $10 \mathrm{kHz}$ and centered around 193.4 THz are divided into odd and even channels, and independently modulated with two I/Q modulators driven by a 4-channel 64 GSa/s arbitrary waveform generator (AWG) to produce a PS 1024-QAM WDM signal. Two symbol rates and frequency grids are used in the experiment, i.e. either $12 \mathrm{GBd}$ on a $12.5-\mathrm{GHz}$ grid or $24.5 \mathrm{GBd}$ on a $25-\mathrm{GHz}$ grid. The modulated signals are combined by a 3-dB coupler, amplified by an EDFA and then passed through a delay-and-add polarization emulator to generate polarization-multiplexed signals. Thirty de-correlated SDM channels are generated using splitters, amplifiers and delays (at least $2.5 \mathrm{~ns}$ between SDM channels) and then launched into a 9.6-km heterogeneous single-mode 30-core fiber through a 3Dwaveguide based fan-in device. The launched power for each core is between 4 and $8 \mathrm{dBm}$ accounting for loss-variations in the fan-in/fanout from $5 \mathrm{~dB}$ to $8 \mathrm{~dB}$.

The single-mode 30-core fiber has four different types of cores to realize a high-density core arrangement with a low cross-talk of $<-50 \mathrm{~dB}$ after $9.6 \mathrm{~km}^{11}$, which can support such a large constellation QAM signal transmission without MIMO processing. The 30 cores are arranged within the limited cladding diameter of $228 \mu \mathrm{m}$, with $A_{\text {eff }}$ of $\sim 80 \mu \mathrm{m}^{2}$. At the output of the 30-core fiber, the 30 spatial channels are demultiplexed using another 3D-waveguide based fan-out device. The demultiplexed spatial channels are then amplified; one WDM channel at a time is filtered out and detected with a polarization-diversity coherent receiver followed by a digital sampling oscilloscope (DSO, 80 $\mathrm{GS} / \mathrm{s}, \quad 33 \mathrm{GHz}$ ) and offline digital signal processing.

\section{Probabilistic shaping, FEC and DSP}

The user data is encoded by a convolutional turbo code, and then many-to-one bit-to-symbol mapping is performed in order to achieve an optimized probability mass function (PMF) of the QAM symbols ${ }^{8}$. With many-to-one labeling, in contrast to standard QAM with unique Gray mapping, multiple bit strings are mapped to the same constellation symbol, making it appear more often on average (see histogram on Fig. 1). The resulting ambiguities are resolved by the decoder. The net data rate of this scheme is controlled by properly puncturing the turbo code to the desired SD-FEC overhead. Data rates of $5,5.5$ and 6 information bits/QAM symbol are used (see Table 1). The PS QAM symbols are then uniformly interleaved with 10\% QPSK symbols, which carry 2 bits per symbol, resulting in a hybrid modulation. Finally, a frame alignment sequence (FAS) is added to the beginning of the sequence, pulse shaping is performed (square root raised cosine, roll-off 0.01 ), and the waveform is sent to the AWG.

At the receiver, actual soft decision demapping and turbo decoding is performed. More than 300 blocks of 6600 (6000 QAM + 600 QPSK) symbols are transmitted and decoded in each case, making the number of decoded information bits $>10^{7}$ in all cases and thereby BER values above $10^{-5}$ reliable. Consequently, when no errors are measured, the BER is reliably below $10^{-5}$. An outer HD-FEC ${ }^{12}$ is then assumed with overhead $0.8 \%$ and decoding threshold of $5 \times 10^{-5}$, which can correct the remaining errors and bring the BER below $10^{-15}$.

\section{Experimental results}

Fig. 2 shows the achieved mutual information (MI, bits/QAM symbol) ${ }^{8}$ and achieved spectral efficiency (SE, bits/s/Hz/polarization/core) after the 30-core fiber transmission of all the WDM and SDM channels for the cases of 12-GBd PS PDM-1024-QAM signal on a 12.5-GHz grid and 24.5-GBd PS PDM-1024-QAM signal on a $25-\mathrm{GHz}$ grid. The $\mathrm{MI}$ gives an upper bound on the achievable SE using ideal SD-FEC decoding.

Tab. 1: Summary of the main results.

\begin{tabular}{|c|c|c|c|c|c|c|c|c|c|}
\hline & \multirow{2}{*}{$\begin{array}{l}\text { HD-FEC } \\
\text { overhead }\end{array}$} & \multirow{2}{*}{$\begin{array}{l}\text { QPSK } \\
\text { hybrid }\end{array}$} & \multicolumn{3}{|c|}{$\begin{array}{c}\text { Tested input data rate } \\
\text { (shaping) [bits/QAM symbol] }\end{array}$} & \multirow{2}{*}{$\begin{array}{c}\text { Achieved } \\
\text { average } \\
\text { SE } \\
\text { [bit/s/Hz/ } \\
\text { pol/core] }\end{array}$} & \multirow{2}{*}{$\begin{array}{c}\text { Achieved } \\
\text { average MI } \\
\text { (hybrid) } \\
\text { [bits/symbol] }\end{array}$} & \multirow{2}{*}{$\begin{array}{c}\text { Achieved } \\
\text { aggregated } \\
\text { SE } \\
{[\mathrm{bit} / \mathrm{s} / \mathrm{Hz}]}\end{array}$} & \multirow{2}{*}{$\begin{array}{c}\text { Achieved } \\
\text { aggregated data } \\
\text { rate per frequency } \\
\text { channel [Tbps] }\end{array}$} \\
\hline & & & \multicolumn{3}{|c|}{$\begin{array}{l}\text { Tested input net SE } \\
\text { [bit/s/Hz/pol/core] }\end{array}$} & & & & \\
\hline \multirow{2}{*}{$\begin{array}{c}12 \text { Gbaud @ } \\
12.5 \mathrm{GHz}\end{array}$} & \multirow{4}{*}{$0.8 \%$} & \multirow{4}{*}{$10 \%$} & 5 & 5.5 & 6 & & & & \\
\hline & & & 4.502 & 4.935 & 5.268 & 4.3050 & (5.031 & 257.010 & 0.122 \\
\hline \multirow{2}{*}{$\begin{array}{l}24.5 \text { Gbaud } \\
\text { @ } 25 \mathrm{GHz}\end{array}$} & & & 5 & 5.5 & 6 & \multirow{2}{*}{4.6712} & \multirow{2}{*}{5.0901} & \multirow{2}{*}{280.272} & \multirow{2}{*}{7.006} \\
\hline & & & 4.596 & 5.038 & 5.479 & & & & \\
\hline
\end{tabular}


(a)

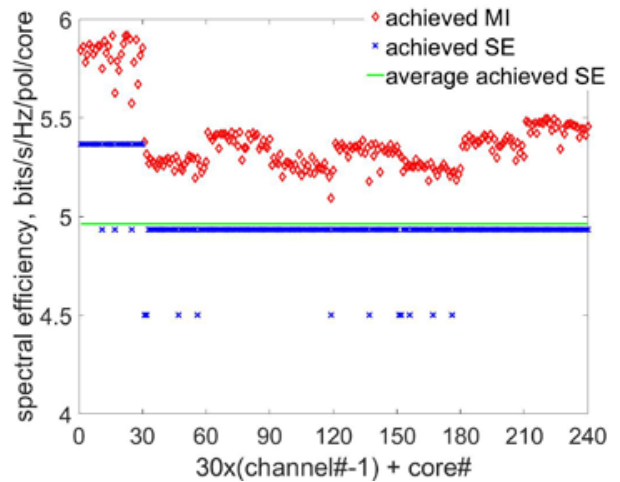

(b)

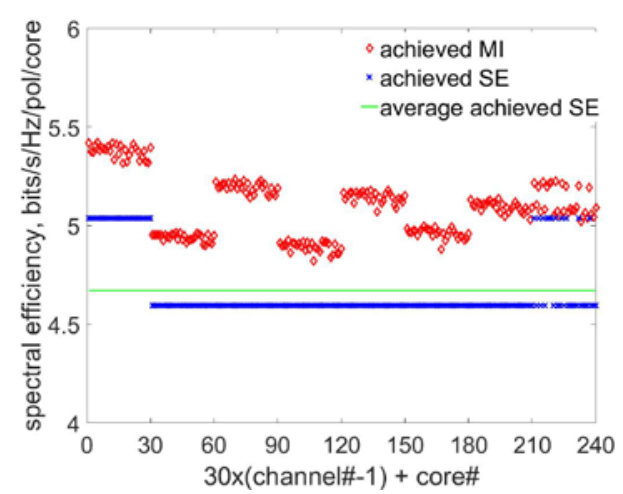

Fig. 2: Achieved mutual information (MI, bits/symbol) and achieved spectral efficiency (SE, bits/s/Hz) using the employed non-ideal SD-FEC decoding after the 30-core transmission of all the WDM and SDM channels for the cases of 12-Gbaud PS PDM-1024-QAM signal on 12.5-GHz grid (a) and 24.5-Gbaud PS PDM-1024-QAM signal on $25-\mathrm{GHz}$ grid (b). Channel\# corresponds to the WDM channels (from 1 to 8 ) and core\# corresponds to the cores of the 30-core fiber (from 1 to 30).

We also performed actual SD-FEC decoding, and the experimentally achieved SE based on our non-ideal codes is also shown. Due to the OSNR variations between the WDM channels and loss variations in the different cores of the 30-core fiber, the transmission performances of the WDM/SDM channels are different. Adaptive rates and SEs are used to achieve error-free performance for different WDM/SDM channels without changing the modulation format. For the 12-GBd PS PDM1024-QAM signal on a $12.5 \mathrm{GHz}$ grid, the achieved average $\mathrm{MI}$ is 5.39 bits/QAM symbol and the achieved average SE with employed FEC decoding is $4.96 \mathrm{bit} / \mathrm{s} / \mathrm{Hz} /$ pol/core, resulting in an aggregated spectral efficiency of 297.8 bit/s/Hz. For the 24.5-GBd PS PDM-1024-QAM signal on a $25 \mathrm{GHz}$ grid, the achieved average $\mathrm{MI}$ is 5.09 bits/QAM symbol and the achieved aggregated net data rate per frequency channel is 7.006 Tbit/s, as summarized in Table 1.

\section{Conclusion}

We have demonstrated adaptive rates and SES in WDM/SDM transmission over a 9.6-km 30core fiber using probabilistically shaped PDM1024-QAM signals with up to 7 Tbit/s per frequency channel. Actual SD-FEC decoding was employed to obtain error-free performance, and owing to the shaping gain we achieved record-high aggregate spectral efficiencies of up to $297.8 \mathrm{bit} / \mathrm{s} / \mathrm{Hz}$ for SM-MCF transmission systems.

\section{Acknowledgments}

DNRF research center of excellence SPOC, ref DNRF123 and EU-Japan project on "Scalable And Flexible optical Architecture for Reconfigurable Infrastructure (SAFARI)" commissioned by the MIC, Japan and EC Horizon 2020.

\section{References}

[1] H. Takara et al., "1.01-Pb/s (12 SDM/222 WDM/456 $\mathrm{Gb} / \mathrm{s}$ ) Crosstalk-managed Transmission with $91.4-\mathrm{b} / \mathrm{s} / \mathrm{Hz}$ Aggregate Spectral Efficiency," Proc. ECOC, Th.3.C.1, (2012).

[2] B. J. Puttnam et al., " $2.15 \mathrm{~Pb} / \mathrm{s}$ transmission using a 22 core homogeneous single-mode multi-core fiber and wideband optical comb," Proc. ECOC, PDP.3.1 (2015).

[3] D. Soma et al., "2.05 Peta-bit/s super-nyquist-WDM SDM transmission using 9.8-km 6-mode 19-core fiber in full C band," Proc. ECOC, PDP.3.2 (2015).

[4] T. Mizuno et al., "32-core Dense SDM Unidirectional Transmission of PDM-16QAM Signals Over 1600 km Using Crosstalk-managed Single-mode Heterogeneous Multicore Transmission Line," Proc.OFC, Th5C.3 (2016).

[5] M. Yoshida et al., "1024 QAM, 7-core (60 Gbit/s x 7) fiber transmission over $55 \mathrm{~km}$ with an aggregate potential spectral efficiency of $109 \mathrm{bit} / \mathrm{s} / \mathrm{Hz}$," Opt. Express, 23(16), p. 20760 (2015).

[6] D. Qian et al., "698.5-Gb/s PDM-2048QAM transmission over 3km multicore fiber," Proc. ECOC, Th.1.C.5 (2013).

[7] D. Soma et al., "665 and $947 \mathrm{~b} / \mathrm{s} / \mathrm{Hz}$ Ultra-highly Aggregate-Spectral-Efficient SDM/WDM Transmission over 6-Mode 19-Core Fibre Using DP-16QAM/64QAM Signals," Proc. ECOC, Th.3.C.2 (2016).

[8] M. P. Yankov, et. al. "Constellation shaping for WDM systems using 256QAM/1024QAM with probabilistic optimization", J. Lightwave Technol., 34(2), 2016.

[9] S. Chandrasekhar et al., "High-spectral-efficiency transmission of PDM 256-QAM with Parallel Probabilistic Shaping at Record Rate-Reach Trade-offs," Proc. ECOC, Th.3.C.1 (2016).

[10] F. Buchali et al., "Experimental demonstration of capacity increase and rate-adaptation by probabilistically shaped 64-QAM," Proc. ECOC, PDP.3.4 (2015).

[11] Y. Amma et al., "High-density Multicore Fiber with Heterogeneous Core Arrangement," Proc. OFC, Th4C.4 (2015).

[12] D. S. Millar, et. al, " Design of a $1 \mathrm{~Tb} / \mathrm{s}$ superchannel coherent receiver", J. Lightwave Technol., 34(6), 2016. 